\title{
Clinical, histological and radiological findings of invasive ductal carcinoma in a pregnant woman
}

\begin{abstract}
Pregnancy-associated breast cancer is defined as a breast cancer diagnosed during pregnancy or within one year postpartum. It is an under-studied entity, despite its increased incidence due to the tendency to extend the age of the first gestation. The major issue regarding breast cancer in pregnant women is the delay in diagnosis, both due to glandular and ductal hyperplasia typical of pregnancy, as well as the physician's uncertainty about the diagnostic approach, fearing harmful effects on the fetus, thus leading to a worse prognosis. We report the case of a pregnant patient with invasive ductal carcinoma, suspected by palpation during physical examination and confirmed after histological study of the fragment obtained by ultrasound-guided core biopsy, with subsequent staging with magnetic resonance imaging The purpose of this report was to discuss the correct investigative management of a case of invasive ductal carcinoma associated with pregnancy, aiming at reducing morbidity and mortality.
\end{abstract}

Keywords: breast cancer, pregnancy, fetus, mammary ultrasonography, magnetic resonance imaging
Volume 6 Issue 5 - 2019

\begin{abstract}
Andressa Almeida de Noronha,' Ritamaris de Arruda Régis, ${ }^{2}$ Ana Rita Régis Borges ${ }^{3}$

'Medical Student, Federal University of Mato Grosso, Brazil ${ }^{2}$ Radiologist, Júlio Muller University Hospital, Brazil

${ }^{3}$ Medical Student, University Center of Várzea Grande, Brazil
\end{abstract}

\begin{abstract}
Correspondence: Andressa Almeida de Noronha, Medical student, Federal University of Mato Grosso, Av Fernando Correa da Costa 2367, Cuiabá, Mato Grosso, Brazil, Tel +5565999044106, Email andressa.noronha@hotmail.com
\end{abstract}

Received: October 17, 2019| Published: October 23, 2019
Abbreviations: $\mathrm{PABC}$, pregnancy-associated breast cancer; FNA, fine-needle aspiration puncture; MRI, magnetic resonance imaging

\section{Introduction}

Pregnancy-associated breast cancer (PABC) is defined as that diagnosed during pregnancy or within one year after delivery. ${ }^{1}$ Its incidence is estimated at 1 for every 3.000 pregnancies, and studies point to an increase in incidence due to the tendency to extend the age of the first pregnancy. ${ }^{2}$ Among women under 45 years, the PABC rate ranges from 2.6 to $6.9 \%$ of cases. ${ }^{1}$ In sporadic cases of pregnancyassociated breast cancer, the most common histological type involved is the invasive ductal carcinoma, which accounts for 78 to $88 \%$ of cases. ${ }^{1}$ The major issue regarding breast cancer in pregnant women is the delay in diagnosis, often exceeding six months, leading to a worse prognosis. ${ }^{2}$ On average, only $1 / 3$ of PABC's are diagnosed during pregnancy, with the other $2 / 3$ diagnosed during the postpartum period. ${ }^{1}$ This delay is due both to glandular and ductal hyperplasia typical of pregnancy, which generates an irregular breast that is difficult to evaluate on physical examination, as well as the physician's uncertainty about the diagnostic approach, fearing harmful effects on the fetus. ${ }^{2-4}$ Therefore, this case report will discuss the diagnostic approach of an invasive ductal breast cancer in a pregnant woman, focusing on the role of imaging tests, aiming at reducing morbidity and mortality.

\section{Case report}

A 33-year-old female primiparous woman, in the second trimester of pregnancy, reports a recent painless lump in the right breast. On physical examination, left breast without changes, there is the presence of a solid and irregular lump in the right breast at $11 \mathrm{am}$, measuring approximately $4.1 \mathrm{~cm}$. The mammary ultrasound (Figure 1) showed an irregularly shaped hypoechoic solid nodule with spiculated margins, located in the right breast, between $10-11 \mathrm{hs}, 6 \mathrm{~cm}$ from the areolar border and measuring $4.1 \times 2.9 \mathrm{~cm}$, BI RADS 4B. A core biopsy was performed and four formalin fixed fragments for histological analysis were sent. Microscopic diagnosis of high-grade/3 mammary duct infiltrative carcinoma (Scarff-Bloom-Richardson, modified by Elston and Ellis) affecting all sampled fragments, corresponding to about $60 \%$ of the sample. No lymphatic or blood vascular infiltration was observed.
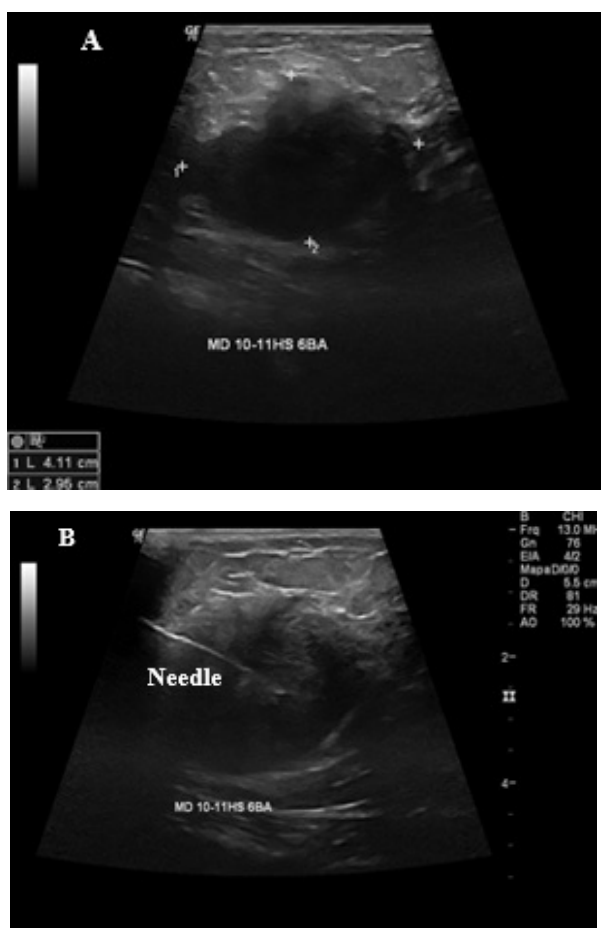

Figure I (A) Breast ultrasound showing an irregularly shaped solid node (crosses) with spiculated margins, located in the right breast. (B) core-biopsy guided by breast ultrasound showing the needle inside the lump 
Proceeded to magnetic resonance staging (Figure 2), with a hyperintense nodule observed in the T1-weighted sequence and heterogeneous in the T2-weighted sequence STIR, oval-shaped and lobulated margins, located in the lateral superior quadrant of the right breast, between $10-11 \mathrm{hs}$, with intense post-contrast enhancement in washout type III pattern and measuring about $6.1 \times 4.6 \mathrm{~cm}$, BIRADS 6; and three right armpit adenomegalies measuring 1.7, 1.9 and 2.5 cm respectively.

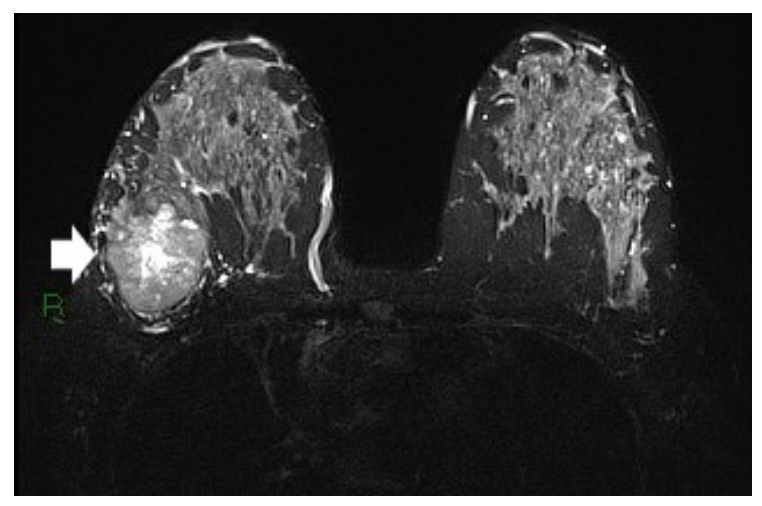

Figure 2 Magnetic resonance of the breasts in the post-contrast dynamic sequence, axial plane, showing right breast lump with enhancement (arrow).

\section{Discussion}

The definitive pathophysiological mechanisms associated with the onset of PABC remain unclear, although some hypotheses have been proposed. ${ }^{1}$ The suggested and possibly non-excluding mechanisms refer to hormonal participation inducing cell proliferation and tumorigenesis, in addition to stimulation of the malignant cells that had already developed in the tissue; immune changes in pregnancy which could lead to cellular immunosuppression, immune tolerance and enhanced inflammatory responses. ${ }^{1}$

It is known that breast cancer in pregnant women has a delayed diagnosis, being the average diagnostic delay around six months, ranging from 2 to 15 months, leading to a worse prognosis, since the risk of lymph node involvement increases in $0.028 \%$ /day based on a period of 130 days for cell duplication. ${ }^{2-4}$ On average, only $1 / 3$ of PABC are diagnosed during pregnancy, and the other $2 / 3$ diagnosed during the postpartum period, the latter being associated with a worse prognosis. ${ }^{1,6}$ The main causes for the delayed diagnosis are the glandular and ductal hyperplasia typical of pregnancy, which generates an irregular breast which is difficult to evaluate on physical examination, and the physician's uncertainty about the correct diagnostic approach, fearing harmful effects to the fetus. ${ }^{2}$ The suspicion of the breast cancer at the patient reported was through mass palpation on physical examination, and the mammary ultrasonography followed by core biopsy was used as the initial investigation.

Mammary ultrasound has sensitivity between 93 and 100\% and a negative predictive value of $100 \%$ in the diagnosis of pregnancyassociated breast cancer. It is a non-invasive exam that allows the immediate execution of a guided biopsy in suspicious masses at any gestational age. ${ }^{3-5}$ Core biopsy is preferred over fine-needle aspiration puncture (FNA) because it is a more sensitive and specific examination than FNA in pregnant breasts. ${ }^{4}$ The breast ultrasound revealed an irregularly shaped hypoechoic solid nodule and spiculated margins with microscopic findings through core biopsy compatible with infiltrative ductal carcinoma of the breast, confirming the diagnosis of breast cancer. A digital bilateral mammography would also be an acceptable exam to be performed from the fetal radiation exposure perspective, because when associated with appropriate shielding, it has a fetal irradiation of approximately $0.004 \mathrm{~Gy}$, being the minimum value to induce negative effects to the fetus $100 \mathrm{mGy} .{ }^{4,7}$ However, it is noteworthy that the higher the radiological density of the breast, the lower the sensitivity of the mammogram, therefore the choice of breast ultrasound on this study. ${ }^{2,4}$

Due to the need for additional studies for the correct staging of the disease, aiming at the correct treatment planning, a breast magnetic resonance imaging was performed and three right-sided adenomegalies were found, suggesting local lymph node involvement. To date, magnetic resonance imaging (MRI) has not been shown to have any adverse effects on human fetus, confirmed by studies which followed until 9 years after fetal exposure. ${ }^{7}$ The potential theoretical teratogenic effects of MRI result from the static magnetic field, which can potentially alter cell migration, proliferation and differentiation and the use of gadolinium which can cross the placenta. ${ }^{4,7}$ However, the risk of an incorrect staging is greater than the potential damage to the fetus and can then be performed after the first trimester of pregnancy, i.e. after organogenesis, if there is any doubt concerning the staging. ${ }^{3,4}$ Treatment should be as similar as possible to that offered to non-pregnant women. ${ }^{8}$ Surgical treatment is considered safe to be performed at any trimester of pregnancy, with mastectomy being the surgery of choice. ${ }^{89}$ Whether to perform or not a sentinel lymph node biopsy during the procedure remains without consensus in the literature. ${ }^{8}$ Chemotherapy can also be performed after the first trimester of pregnancy, taking care to avoid certain chemotherapeutic drugs such as methotrexate. ${ }^{9}$ Radiotherapy, hormone therapy and use of monoclonal antibodies are contraindicated during pregnancy. ${ }^{8,9}$

\section{Conclusion}

Pregnancy-associated breast cancer is a challenging disease, due to its difficult diagnosis, despite the progressive incidence. Correct investigative management is essential in cases of suspected breast cancer associated with pregnancy, aiming at reducing morbidity and mortality. The first line of investigation should be mammary ultrasound because it is a safe and highly sensitive and specific imaging exam for the diagnosis of breast cancer in pregnant women, which enables the performance of a guided core-biopsy if there is a suspicious lump. Other complementary imaging tests can be performed for the correct staging of the disease, always assessing the risk/benefit for the patient.

\section{Acknowledgments}

None

\section{Conflicts of interest}

Author declares that there is no conflict of interest.

\section{References}

1. Ruiz R, Herrero C, Strasser-Weippl K, et al. Epidemiology and pathophysiology of pregnancy-associated breast cancer: A review. Breast. 2017;35:136-141.

2. Vinatier E, Merlot B, Poncelet E, et al. Breast Cancer during pregnancy. Eur J Obstet Gynecol Reprod Biol. 2009;147(1): 9-14.

3. Ayyappan AP, Kulkarni S, Crystal P. Pregnancy-associated breast cancer: spectrum of imaging appearances. Br J Radiol. 2010;83(990):529-534.

4. Case AS. Pregnancy-associated Breast Cancer. Clin Obstet Gynecol. 2016;59(4):779-788. 
5. Kakoulidis L, Skagias L, Politi E. Pregnancy associated breast cancer (PABC): aspects in diagnosis. Breast Dis. 2015;35(3):157-166.

6. Lee GE, Mayer EL, Partridge A. Prognosis of pregnancy-associated breast cancer. Breast Cancer Res Treat. 2017;163(3):417-421.

7. Woitek R, Prayer D, Hojreh A, et al. Radiological staging in pregnant patients with cancer. ESMO Open. 2016;1:e000017.
8. Knabben L, Mueller MD. Breast cancer and pregnancy. Horm Mol Biol Clin Investig. 2017;32(1):1868-1891.

9. Cordeiro CN, Gemignani ML. Breast cancer in pregnancy: Avoiding fetal harm when maternal treatment is necessary. Breast J. 2017;23(2):200 205. 